\title{
Moving toward and away from others: Social orientations in emerging adulthood
}

\author{
Nathan A. Jorgensen \\ Brigham Young University - Provo \\ Larry J. Nelson \\ Brigham Young University - Provo, larry_nelson@byu.edu
}

Follow this and additional works at: https://scholarsarchive.byu.edu/facpub

Part of the Other Social and Behavioral Sciences Commons

\section{Original Publication Citation}

*Jorgensen, N. A., \& Nelson, L. J. (2018). Moving toward and away from others: Social orientations in emerging adulthood. Journal of Applied Developmental Psychology, 58, 66-76.

\section{BYU ScholarsArchive Citation}

Jorgensen, Nathan A. and Nelson, Larry J., "Moving toward and away from others: Social orientations in emerging adulthood" (2018). Faculty Publications. 4705.

https://scholarsarchive.byu.edu/facpub/4705 


\title{
Moving toward and away from others: Social orientations in emerging adulthood
}

\author{
Nathan A. Jorgensen*, Larry J. Nelson \\ School of Family Life, Brigham Young University, 2086 JFSB, Provo, UT 84602, United States
}

\section{A R T I C L E I N F O}

\section{Keywords:}

Social interaction

Social withdrawal

Relational/individual well-being

Emerging adulthood

\begin{abstract}
A B S T R A C T
As emerging adults navigate numerous changes to their relationships, the ways in which they connect with and move away from others, or how they are socially oriented, may play an important role in their relational and individual well-being. The current study explored holistic types of social orientations (i.e., social motivations, the self in relation to others, other-directed emotions, and actual behaviors) and how they relate to the quality of close relationships, depression, and substance use in a sample of 787 US emerging adult college students. Results from latent profile analysis suggested five types of social orientations, each showing a distinct pattern of moving toward or away from others and links to varying degrees of relational and individual well-being. This study's consideration of multiple aspects of social orientations not only advances current theoretical models of social interaction, but also has important implications for understanding mechanisms that lead to flourishing and floundering in emerging adulthood.
\end{abstract}

\section{Introduction}

With the many transitions that can take place during emerging adulthood (e.g., leaving the parental home, entering college or workforce, developing romantic relationships), young people frequently face the challenge of navigating complex changes to their relationships and social interactions (Barry, Madsen, \& DeGrace, 2016; Padilla-Walker, Memmott-Elison, \& Nelson, 2017). Thus, researchers have recognized the importance of understanding how emerging adults connect with and relate to others (Bowker, Nelson, Markovic, \& Luster, 2014; Nelson, 2013), as these connections have clear ties to relational and individual well-being (Padilla-Walker et al., 2017). One of the primary lenses through which researchers have previously examined the extent to which individuals orient themselves toward or away from others is via social approach and avoidance motivations (Asendorpf, 1990). This model has made significant contributions to the field of social development, demonstrating the existence of various social subtypes and how they relate to well-being (see Coplan \& Armer, 2007). However, recent work has challenged the idea that types of sociality can be fully captured by social motivations alone (Bowker, Stotsky, \& Etkin, 2017), suggesting that there may be other important factors that influence how people are oriented toward or away from others.

In this paper, the term "social orientations" is proposed to represent a broad and holistic way of understanding the various ways in which emerging adults might move toward or away from others. Specifically, in addition to social motivations, how people view the self in relation to others, how people feel and think about others, and how people behave socially have yet to be explored together as aspects of social orientations. Therefore, the overall aim of this study was to present social orientations as a meaningful construct for understanding social adjustment and well-being in emerging adulthood. To accomplish this, the first purpose of the study was to employ a person-centered analysis (i.e., latent profile analysis) to examine variations in the extent to which individuals orient themselves toward or away from others in their social motivations, self-processes in relation to others, other-directed emotions, and actual social behaviors via latent profile analysis. The second purpose of this study was then to determine the extent to which socialorientation groups differed on indices of relational (i.e., relationship quality with best friends, romantic partners, and parents) and individual well-being (i.e., depression and substance use). Because no known study has taken a holistic approach to studying social orientations, a secondary aim of this study was to advance theory by synthesizing several related areas of research under the label of social orientations.

\section{The importance of social orientations in emerging adulthood}

The transition from adolescence to adulthood presents a truly

\footnotetext{
* Corresponding author.

E-mail addresses: njorgensen@unc.edu (N.A. Jorgensen), larry_nelson@byu.edu (L.J. Nelson).
} 
unique time period in terms of how the self relates to others. On the one hand, emerging adults become more independent and experience increased autonomy in their life choices, including when and how they will interact with others (e.g., social lifestyle, type of education/career, living alone or with roommates, etc.). On the other hand, they also face increased exposure to novel social environments and interactions with new people that are often beyond their control (e.g., coworkers, roommates, classmates, etc.). Thus, emerging adults must make choices about how to navigate complex developmental changes in the nature of their relationships with others, including choices about maintaining old relationships and establishing new ones. Of course, with increased independence, they are also presented with the opportunity to move away from others by not maintaining or pursuing relationships. Although the extent to which they move toward or away from others is more within their control than ever before, the consequences of those choices (e.g., choosing not to engage in social settings such as work or school) may be greater than ever before as well. Indeed, they may be predictive of various aspects of flourishing (e.g., healthy relationships with others, positive mental health) and floundering (e.g., internalizing problems such as depression, externalizing problems such as risky substance use) in emerging adulthood (Nelson \& Padilla-Walker, 2013). In sum, there is a need to better examine social orientations (i.e., the various processes that underlie choices to move toward and away from others) in emerging adults, as they may have significant implications for individual and relational well-being in the third decade of life.

Social orientations can offer a new approach to understanding relationships in emerging adulthood. Most previous research on this topic has focused on factors such as formation processes (i.e., similarities that bring people together) and relationship qualities (e.g., intimacy, commitment, conflict; Barry et al., 2016; Shulman \& Connolly, 2016), but there has been less attention given to individual differences that might precede both the formation and quality of these relationships. In other words, the extent to which one simply engages in social interactions (i.e., a precursor to forming relationships) needs greater examination in emerging adulthood. A deeper understanding of social orientations may provide meaningful insight into some of these individual differences. For example, some orientations may promote more social interactions and, in turn, the formation of more and better-quality relationships than other orientations. Additionally, once relationships form, social orientations may then act as a personal characteristic that promotes (e.g., balances the self and others) or inhibits (e.g., creates dependence on others) healthy relational functioning.

Taken together, neither studies of the self nor studies of relationships alone can capture an important aspect of emerging adults' social lives. Given that emerging adults' choices to interact or not may be a precursor to so many aspects of flourishing or floundering, it would be important to examine how individuals see themselves in relation to others or, in other words, to examine the various ways in which individuals (i.e., the self) think, feel, and behave in relation to others. As such, if individuals differ in their social orientations toward or away from others, they should also differ in indices of flourishing and floundering at relational and individual levels. Previous research suggests that this may indeed be the case. For example, social motivations that orient people away from others are related to peer and relational problems (Bowker et al., 2014) and depression (Nelson, 2013). Overinvolvement of others in self-evaluations is related to relationship insecurity (Fitzsimons \& Anderson, 2013), anxiety and depression (Harter, 2012), and increased susceptibility to social pressures, which can include negative behaviors such as substance use (Litt, Stock, \& Gibbons, 2015). Although these studies each only addressed one aspect of social orientations, they do offer initial evidence that social orientations may be able to predict relational and individual well-being in emerging adults. In summary, gaining a deeper understanding of how emerging adults are socially oriented may help uncover more reasons why some flourish and some flounder in the third decade of life, which can have important implications not only for the immediate lives of emerging adults, but also for laying foundations for success as they transition into adulthood.

\section{Theoretical challenges: social motivations and social withdrawal}

In order to more fully understand social orientations, however, there are several challenges with existing theoretical and methodological approaches that must first be addressed. One of the primary ways researchers have previously examined social orientations is the social motivations model (Asendorpf, 1990). This model describes several states according to individuals' internal motivations to approach and avoid social interaction, namely sociability (high approach, low avoidance), shyness (high approach, high avoidance), avoidance (low approach, high avoidance), and unsociability (low approach, low avoidance). These states reflect several cognitive (i.e., preferences for social interaction or solitude) and emotional (e.g., enjoyment, fear, anxiety) processes that relate to how individuals are oriented toward others. These processes, albeit limited, have been useful for both identifying certain types of social orientations and demonstrating how these orientations are differentially associated with indices of wellbeing. In emerging adulthood, shy and avoidant individuals have been shown to be prone to myriad individual and relational challenges (e.g., internalizing problems, low relationship quality; Nelson, 2013). On the other hand, unsociable individuals tend to suffer from far fewer problems, but are still prone to some psychological and health difficulties (i.e., depression and emotional eating; Etkin, Bowker, \& Scalco, 2016; Nelson, 2013). Taken together, these findings demonstrate that emerging adults who are oriented away from others may struggle with both individual and relational well-being, although the degree of these struggles may vary based on individual differences in cognitive and emotional social processes. Thus, social motivations provide a good starting point for understanding overall social orientations, but several shortcomings of this model merit a deeper exploration of other factors that may contribute to social orientations.

First, although the social motivations model can potentially explain reasons for both social engagement and withdrawal, its primary focus has been withdrawal. In other words, there has been much exploration of individual differences in cognitive and emotional processes that lead people away from others, but little inquiry into similar factors that might lead them toward others. Given the social nature of human beings (Baumeister \& Leary, 1995) and that most emerging adults tend to not be socially withdrawn (Nelson, 2013), an attempt to capture overall social orientations requires consideration of both withdrawal and engagement. Second, motivations offer only a narrow view of the vast cognitive, emotional, and behavioral processes that contribute to how one is oriented toward or away from others. Specifically, past research has demonstrated that in addition to social motivations, several factors contribute to social orientations and predict indices of well-being. These include how people view the self in relation to others, additional other-directed emotions, and actual social behaviors. In the past, however, these have been analyzed separately, and no known study has considered these together as components of overall social orientations.

Furthermore, most previous research has employed variable-centered analyses (e.g., shyness is associated with depression; Etkin et al., 2016). Although these types of findings offer insight into social characteristics that are associated with well-being outcomes, they fail to consider that social orientations may be better conceptualized as patterns across a number of social characteristics rather than simply the social variables themselves. To this end, there have been some personcentered analyses aimed at identifying distinct types of sociality across more than one variable. For example, rather than treat shyness and sociability as conflicting constructs, Cheek and Buss (1981) recognized that some people can be both shy and sociable, and created four distinct groups based on these variables: shy-sociable, shy-unsociable, unshysociable, and unshy-unsociable. Other studies, based on the social motivations model, separated individuals into shy, avoidant, and 
unsociable groups based on their scores on related measures (Nelson, 2013; Nelson, Coyne, Howard, \& Clifford, 2016). Although all of these studies demonstrated that types of sociality can be linked to specific indices of adjustment (e.g., internalizing problems, self-esteem), two issues indicate the need for further study. First, these studies used mean-splitting methods to conduct person-centered grouping, or assigning people into groups based on high vs. low scores on various variables. More sophisticated methods, such as latent profile analysis, offer more exact classification of groups based on statistically identified patterns across variables rather than simply scores that are higher or lower than the mean. Second, these studies are limited in their coverage of social characteristics that contribute to how people are socially oriented (e.g., only examine cognitions related to desires to approach and/or avoid others). As will be reviewed in the following sections, there are several important aspects of social orientations that have not yet been integrated into one model. Thus, the latent profile analysis approach taken in this study not only offers more precise methods of identifying groups, but also allows for consideration of a number of important social characteristics (i.e., how people view the self in relation to others, additional other-directed emotions, and actual social behaviors) that contribute to social orientations.

\section{The self in relation to others}

Of the several definitions of "self" used in psychological research (see Leary \& Tangney, 2012), two are especially applicable to social orientations. Specifically, the self is considered as both beliefs about oneself and the individual's executive agent (i.e., the agent that regulates behavior and decision-making).

\section{Beliefs about oneself}

At a basic level, beliefs about the self may act as an initial step in approaching or avoiding social interaction, such as confidence (or lack thereof) to interact with other people (Harter, 2012). However, perhaps more important for understanding social orientations are the processes by which these beliefs are formed. Beliefs about the self develop as individuals receive and interpret information pertaining to themselves. Although this includes some information that can be objective and independent of other people (e.g., personal observations, abilities, performance, etc.), the majority of self-relevant information comes from others, both directly and indirectly (Harter, 2012). Processes by which this occurs include explicit feedback from others (e.g., "You did very well at this"), social comparison (e.g., "I am better than others at this"), and reflected appraisals (e.g., "I bet others think I am bad at this").

Individuals who frequently engage in social comparison can be considered socially oriented toward others, as reflected by high interest in and concern about the evaluations of others. However, frequent social comparers also tend to face relational and personal struggles, such as being influenced by social pressures (Litt et al., 2015), having difficulty making meaningful connections (Yang, 2016), and feeling uncertain of themselves (Buunk \& Gibbons, 2006). Thus, the extent to which individuals involve others (via comparison) in the development of the self may be an important indicator of their social orientation. Reflected appraisals, or perceptions of what others think about the individual, are frequently observed as self-consciousness (e.g., concern for what others think, fear of negative evaluation). Although self-consciousness can be beneficial for adherence to social norms and is quite common during adolescence and emerging adulthood (Harter, 2012), in excess it can lead to increased anxiety, feelings of inferiority, and relationship insecurity (Fitzsimons \& Anderson, 2013). Thus, inclusion of others in the self at an appropriate level may orient individuals toward others and, in turn, be tied to individual and relational well-being. However, at excessive levels it may orient them away from others and thereby contribute to internalizing and relationship difficulties. Hence, in addition to social motivations, this study also examined the extent to which self-evaluations (i.e., self-esteem, social physique) and the inclusion of others in those evaluations (i.e., fear of negative evaluation, social comparison) contribute to different types of social orientations.

\section{The self as executive agent: self-regulation}

Up to this point, we have reviewed various motivations, cognitions (e.g., self-evaluations, social comparison), and emotions (e.g., anxiety, fear, self-conscious emotions) that frequently arise during social interactions and how these each contribute to social orientations. A question remains of how individuals may react to and deal with these experiences differently. Hence, another aspect of the self deserves attention, namely the self as executive agent, or one's ability to exercise selfcontrol, self-regulation, and decision-making (Leary \& Tangney, 2012). In addition to being present in a wide range of individual thoughts and behaviors, the executive self also plays a role in interpersonal behaviors and relationships (Baumeister \& Vohs, 2012), and can thus be considered another part of how the self relates to others. As reviewed above, social interaction often involves uncomfortable and undesirable feelings (e.g., anxiety, fear, self-consciousness). In the presence of social fears and anxieties, various forms of dysregulation (e.g., emotional, physiological, attentional) predict shy and withdrawn behaviors (Eisenberg, Fabes, \& Murphy, 1995). This suggests that when people experience social discomfort, the inability to self-regulate may orient them away from others and lead to withdrawn feelings and behaviors. Additionally, high regulatory functioning in adolescence is indicative of relational and individual outcomes such as positive social relationships (for a review, see Farley \& Kim-Spoon, 2014) and low substance use (Wills, Walker, Mendoza, \& Ainette, 2006). Hence, this study also examined emotional self-regulation as an additional important factor that influences social orientations.

\section{Other-directed emotions}

Although social motivations (Asendorpf, 1990) capture some aspects of how people think (e.g., social preferences) and feel (e.g., anxiety, fear) about interacting with others, there are many more other-directed emotions that contribute to social orientations. For example, Buunk and Gibbons (2006) observed that frequent social comparers, in addition to having lower self-evaluations, are also characterized by empathy, sensitivity to others' needs and feelings, and willingness to help. Thus, it may be useful to examine how various other-directed emotions influence moving toward or away from others. Empathy refers to the emotional ability to understand and share the feelings and experiences of others and is a strong predictor of general social behavior (Berger, Batanova, \& Cance, 2015) and prosocial behavior (Laible, Murphy, \& Augustine, 2014), suggesting that it orients people toward others. Empathic concern has been found to buffer against the negative social repercussions of social anxiety (Batanova \& Loukas, 2011), suggesting that empathic concern may orient people toward others even when other processes (i.e., social anxiety) are orienting them away from others, suggesting that it is important to examine how people feel directly toward others as another component of social orientations. Thus, this study also explored the contribution of other-directed emotions (i.e., empathic concern) in different types of social orientations.

\section{Actual social and nonsocial behaviors}

As noted, another weakness of examining social orientations solely from a motivational approach is that there is evidence that even among people who share similar motivations there may be different patterns in actual behavior. For example, two studies found differences in social behavior among people who experience an approach-avoidance motivational conflict (i.e., social anxiety; Kashdan, Elhai, \& Breen, 2008; Kashdan, McKnight, Richey, \& Hofmann, 2009). Many of these socially anxious people displayed the more predictable patterns of behavioral 
inhibition and risk aversion, but others reported higher levels of social activity, including interacting with others and meeting new people, as well as some externalizing and risk-prone behaviors. One defining characteristic of the people in this group is that their social behaviors were strongly motivated by the desire to advance their social status (Kashdan et al., 2008). In other words, despite the fact that some processes (i.e., fear, anxiety) were pulling these people away from others, other processes (i.e., concerns for the social self) seemed to take precedence and orient them toward others, at least behaviorally. Thus, there is evidence, albeit limited, that in addition to cognitions, emotions, and executive function, it is also important to study how people are behaviorally oriented toward or away from people. Therefore, this study also examined actual social behavior (i.e., social involvement) as an additional contributing factor of social orientations.

\section{Current study}

The first purpose of this study was to examine variations in the extent to which individuals orient themselves toward or away from others in their social motivations (i.e., sociability, shyness, unsociability), self-processes in relation to others (i.e., self-esteem, perceptions of physique, fear of negative evaluation, social comparison, emotional self-regulation), other-directed emotions (i.e., empathic concern), and actual social behaviors (i.e., social involvement) via latent profile analysis. Based on the extant literature, it was expected that at least one group would reflect an appropriate balance between others and the self, at least one other group would be overly oriented toward others, and one would be overly oriented away from others. Although these general trends were expected, the nature of latent profile analysis precluded specific hypotheses about the numbers of groups and precise descriptions of these groups.

The second purpose of this study was to then determine the extent to which social orientation groups differed on indices of relational (i.e., relationship quality with best friends, romantic partners, and parents) and individual well-being (i.e., depression and substance use). These specific outcomes were selected because emerging adults experience a variety of changes to their relationships and face tasks that are more focused on individual growth (Barry et al., 2016; Padilla-Walker et al., 2017). Thus, how individuals approach their connections with others (i.e., social orientations) may be closely related to both relational and individual success. Although it was difficult to form specific hypotheses before knowing specifically what groups would be identified from the latent profile analyses, we expected several trends based on the research reviewed above. Specifically, we expected that those who appropriately balanced the self and others would fare the best on relational and individual outcomes. Those who were overly oriented toward others would struggle individually because of their over-dependence on others, but may have moderately positive relationships because of their concern for these relationships. Hence, we expected those who were overly oriented away from others to struggle both individually and relationally.

\section{Method}

\section{Participants}

Participants for this study were drawn from a study of emerging adults entitled "Project READY" (Researching Emerging Adults' Developmental Years). The sample for the current study $\left(M_{\text {age }}=19.60\right.$, $S D=1.84$, range $=18-29$ ) consisted of 787 undergraduate students (544 female). Participants were recruited from four universities across the United States, including the Pacific-West, South, Mid-West, and Mid-Atlantic regions. Most emerging adults were European-American (69\% European-American, 18\% Asian-American, 5\% Latino-American, $3 \%$ African-American, and $5 \%$ other). Thirty-eight percent of participants reported both parents having a bachelor's degree or more, and $71 \%$ reported their parents having a combined income of over $\$ 50,000$ per year.

\section{Procedure}

After receiving institutional review board approval, participants were recruited through faculty's announcement of the study in large, general education undergraduate courses to access a broad range of students. After a brief in-class explanation by professors, interested students later accessed the study website with a class-specific recruitment code and gave their informed consent before beginning the survey. The survey took approximately $45 \mathrm{~min}$ to complete. Most participants received a \$20 Amazon gift code for their participation, while others received extra credit.

\section{Measures}

We created all scale measures using mean scores, with higher scores representing higher levels of that variable, unless otherwise reported. Scores were calculated for each participant that responded to at least one of the items on the scale. Measures used in the latent profile analysis were standardized prior to the analysis. Because some constructs were measured with multiple variables, some variable names are italicized for readability.

\section{Social motivations}

Social approach and avoidance motivations were measured using the Child Social Preference Scale (Coplan, Prakash, O'Neil, \& Armer, 2004) revised for college students (Nelson, 2013). Participants answered 21 questions on a 1 (strongly disagree) to 5 (strongly agree) point scale. Three scales were created for sociability ( $\alpha=.79$; e.g., "I like to be with people"), shyness ( $\alpha=.91$; e.g., "I'd like to hang out with other people, but I'm sometimes nervous to"), and unsociability ( $\alpha=.70$; e.g., "I like spending time alone more than I like spending time with other people"). In past work using this measure (Nelson, 2013), avoidance has been measured as the reversed score of sociability and is thus not presented in this study to avoid repetition.

\section{Beliefs about the self}

Beliefs about the self were assessed using four measures that included both self-evaluations and the extent to which others influence those evaluations. First, participants rated their self-esteem using five questions ( $\alpha=.85$; e.g., "I am happy being the way I am") from the Self Perceptions Profile for College Students (Neeman \& Harter, 1986). Second, participants' evaluations about their own physique were measured with three questions ( $\alpha=.81$; e.g., "In the presence of others, I feel apprehensive about my physique or figure") from the Social Physique Anxiety Scale (Hart, Leary, \& Rejeski, 1989). Third, fear of negative evaluation was assessed using three questions ( $\alpha=.70$; e.g., "I am afraid others will not approve of me") from the Brief Fear of Negative Evaluation Scale (Leary, 1983). Fourth, two questions about social comparison (e.g., "When I am not certain about how well I am doing at something, I usually like to be around others so I can compare myself to them") were taken from the Inclusion of Other in the Self Scale (Aron, Aron, \& Smollan, 1992). The first measure was on a 4-point scale ranging from 1 (not at all true for me) to 4 (very true for $m e$ ), whereas the latter three measures were on a 5-point scale ranging from 1 (strongly disagree) to 5 (strongly agree).

\section{Self-regulation}

Emotional self-regulation was measured using five questions ( $\alpha=.80$; e.g., "I get upset easily") from the emotional self-regulation subscale (Novak \& Clayton, 2001). These questions were on a 5-point scale ranging from 1 (never true) to 5 (always true). On this scale, higher scores represented emotional dysregulation. 


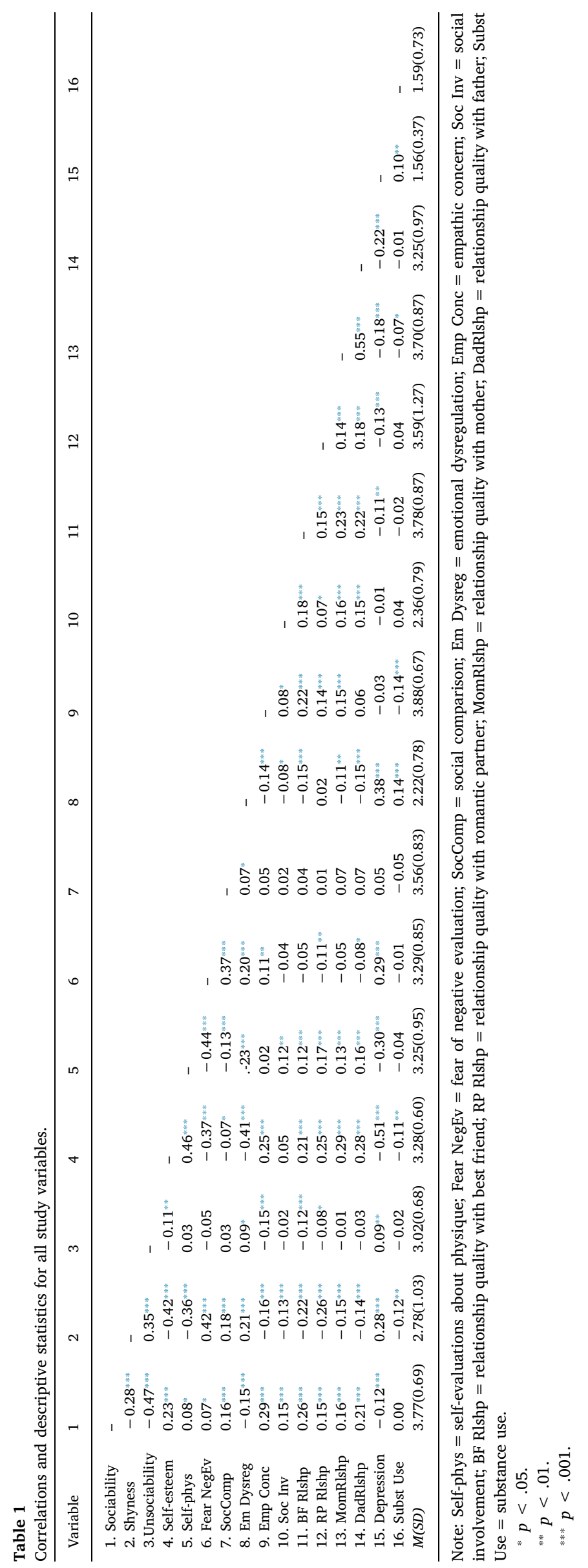




\section{Other-directed emotions}

Other-directed emotions were assessed using five items measuring empathic concern for others ( $\alpha=.79$; e.g., "I am often quite touched by things that I see happen to others"; Davis, 1983). These items were on a 5-point Likert scale ranging from 1 (not like me at all) to 5 (very much like me).

\section{Social behaviors}

Actual social behaviors were measured using five items created for this study ( $\alpha=.69$; i.e., participating in community/civic/church volunteer service, student clubs or associations, spiritual activities with at least one other person, attending campus events, and attending cultural events) that gauged participants' frequency of social involvement, ranging from 0 (none) to 5 (every day or almost every day). Because this study utilized a college student sample, these questions were created to capture types of social involvement common among college student emerging adults.

\section{Relational well-being}

To assess relational well-being, relationship quality (e.g., "How happy are you with the way things are between you and this person?") with participants' best friend, romantic partner, mother, and father were measured using the Social Provisions Questionnaire (Carbery \& Buhrmester, 1998). Participants answered 12 questions about each relationship on a 5-point scale ranging from 1 (little or none) to 5 (the most). Reliabilities were acceptable for all four ratings $(\alpha=.95, .98$, .93 , and .94 , respectively).

\section{Individual well-being}

Two indices of individual well-being were included. Depression levels were assessed using eight questions ( $\alpha=.76$; e.g., "I felt everything I did was an effort") from the original CES-D scale (Radloff, 1977). Questions were answered on a 3-point scale ranging from 1 (never) to 3 (most of the time). Frequency of substance use (i.e., alcohol, tobacco, prescription drugs, and other illegal drugs) was assessed on a 0 (none) to 5 (every day or almost every day) scale $(\alpha=.82)$.

\section{Analysis plan}

A three-step mixture model latent profile analysis (LPA) was conducted in Mplus 7.4 to identify types of social orientations and their associations with indices of relational and individual well-being (Asparouhov \& Muthén, 2014). This form of LPA was chosen for its ability to produce unbiased estimates (Feingold, Tiberio, \& Capaldi, 2014) in identifying groups based on similar patterns of responses to the 10 social orientation variables described above. Larger class separation (i.e., group mean differences on indicator variables) and more indicator variables contribute to greater statistical power to detect the correct number of profiles (Tein, Coxe, \& Cham, 2013). The decision about the correct number of profiles was made according to several model fit indices, including Bayesian information criterion (BIC), the sample size adjusted BIC (SABIC), the Lo-Mendell-Rubin likelihood ratio test (LMR LRT), and the bootstrapped likelihood ratio test (BLRT). The two likelihood ratio tests compare the current model ( $k$ profiles) to the model with one fewer ( $k-1$ profiles). Better model fit is indicated by lower BIC and SABIC values and significant values for the two likelihood ratio tests, although BIC and BLRT are thought to be the best indicators of latent profiles (Nylund, Asparouhov, \& Muthén, 2007; Tein et al., 2013). Finally, profile solutions were compared based on meaningful theoretical interpretation of the profiles. Entropy and average probabilities for most likely profile membership were used to determine if the final model accurately classified individuals into groups, with values closer to 1 indicating more accurate classification of individuals into latent profiles. Additionally, variable-specific entropy contribution values (Asparouhov \& Muthén, 2014) were obtained for each indicator variable to determine the extent to which each variable played a role in classification. The DU3STEP command was used to compare latent profile group means for the distal outcomes of relationship quality with best friend, romantic partner, mother, and father, and individual adjustment outcome of depression. Because the substance use variable was non-normally distributed, group means for this variable were compared using the DCON command, or the Lanza, Tan, and Bray (2013) method, which is robust against non-normal distributions for distal outcomes. Listwise deletion is used for missing data in latent profile analysis in Mplus. This was not problematic, however, since listwise deletion is only used when estimating means for each variable (missing data per variable ranged from zero to seven observations) but does not exclude participants with some missing data in the full analysis.

\section{Results}

\section{Latent profile analysis: social orientations}

Descriptive statistics and correlations between all study variables are displayed in Table 1. Latent profile analyses specifying two to seven profiles were conducted using the social orientation variables and were compared according to model fit indices (see Table 2). BICs decreased noticeably from two to five profiles and then slightly from five to six and six to seven profiles, indicating improvement in model fit as the number of profiles increased. SABICs also indicated improved model fit with more profiles, as they decreased noticeably from two to six profiles and then slightly from six to seven profiles. The LMR LRT $p$-values indicated that a two-profile solution was better than a one-profile solution and a four-profile solution better than three, but did not indicate that more profiles would further improve model fit. The BLRT $p$-values were all less than .001 , indicating that more profiles improved model fit. Because BIC, SABIC, LRT and BLRT indicated that choosing four to six profiles would yield the best model fit, these were compared according to theoretically meaningful interpretations of the groups that were identified. It was determined that the four- and five-profile solutions yielded meaningful groups, whereas the six-profile solution added little substantive distinction between groups. Ultimately, it was decided that the five-profile model offered the most insight into social orientation profiles. One of the profile sizes in this model were noticeably small $(n=12)$, which raises caution about statistical power for ensuing group comparisons. However, there is no fixed rule for class sizes, and it is best to rely on theory and interpretability in making these decisions. As will be discussed below, both the composition and size of these groups are theoretically sound and interpretable. Therefore, the 5-profile model was chosen, but caution should be used when interpreting comparisons with the smallest profile. Entropy for this model was .79, and average probabilities for most likely class membership were .95, $.86, .83, .83$, and .88 , respectively, all indicating good prediction of class membership. Variable-specific entropy contribution values suggest that some variables contributed to the classification of social orientations more than others (sociability $=.29$, shyness $=.40$, unsociability $=.27$, self-esteem $=.30$, physique $=.30$, fear of negative evaluation $=.37$, social comparison $=.21$, dysregulation $=.19$, empathic concern $=.21$, social involvement $=.17$ ).

Table 2

Model fit indices for latent profile analyses.

\begin{tabular}{llllll}
\hline Profiles & BIC & SABIC & LRT & BLRT & Entropy \\
\hline 2 & 21752 & 21653 & $p=.01$ & $p<.001$ & .74 \\
3 & 21627 & 21494 & $p=.11$ & $p<.001$ & .72 \\
4 & 21489 & 21321 & $p=.03$ & $p<.001$ & .7 \\
5 & 21384 & 21181 & $p=.23$ & $p<.001$ & .79 \\
6 & 21330 & 21092 & $p=.36$ & $p<.001$ & .76 \\
7 & 21317 & 21044 & $p=.76$ & $p<.001$ & .77 \\
\hline
\end{tabular}

Note. Group sizes for latent profile solutions are available upon request. 


\section{Latent Social Orientation Profiles}

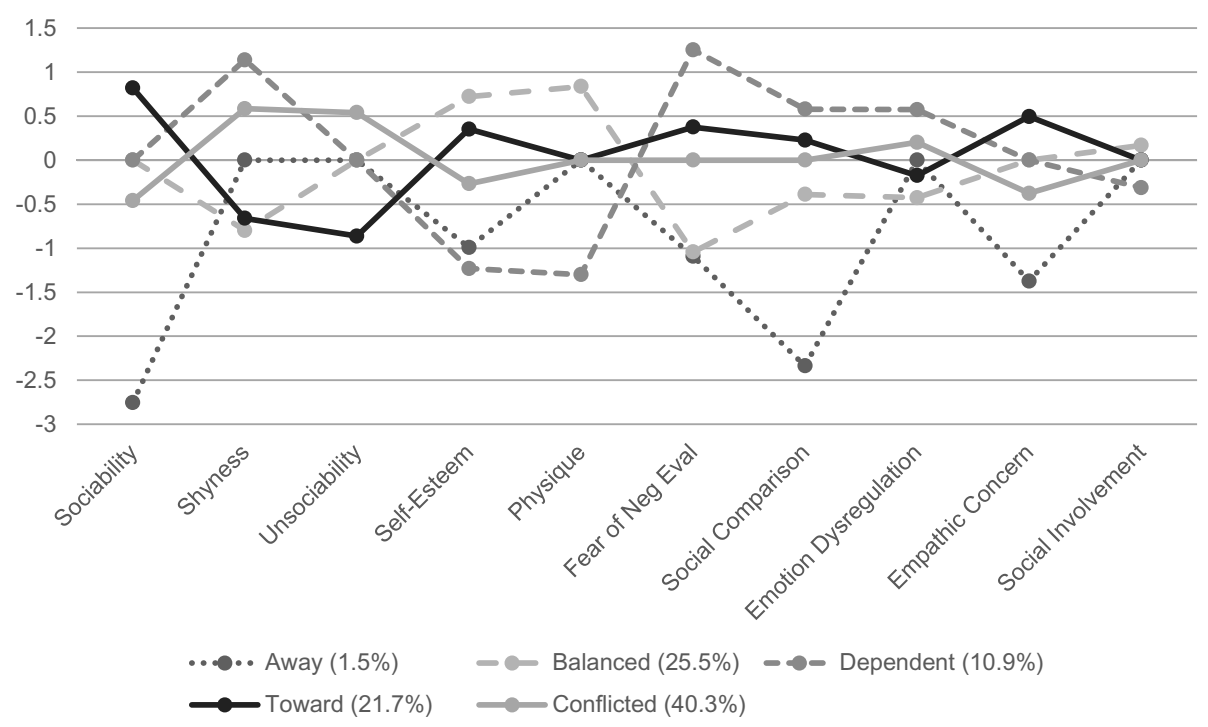

Fig. 1. Response patterns across 10 variables for social orientation profiles.

Estimated group means and comparisons for each social orientation variable are displayed in Appendix and group response patterns in Fig. 1. The first profile ( $n=12,1.5 \%$ of the sample, $75 \%$ male) was characterized by low social approach motivation, poor self-perceptions, little inclusion of others in self-evaluations, and low levels of otherdirected emotions, thus reflecting an overall orientation away from others. Thus, this group was labeled as the Away-from-Others Orientation. The second profile ( $n=201,25.5 \%$ of the sample, $34 \%$ male) was characterized by low social avoidance motivation, high self-perceptions, low inclusion of others in the self, low emotional dysregulation, and higher levels of social behavior. This reflects an overall orientation toward others while balancing the self in relation to others, and was thus given the label of Balanced-toward-Others Orientation. The third profile ( $n=86,10.9 \%$ of the sample, $23 \%$ male) displayed a social motivation conflict (i.e., shyness indicating high approach, high avoidance), low self-evaluations, high inclusion of others in the self, high emotional dysregulation, and lower levels of actual social involvement. Thus, this group appears to be anxious and poorly regulated around others, yet very strongly oriented toward them, and was labeled the Dependent-toward-Others Orientation. The fourth profile $(n=171$, $21.7 \%$ of the sample, $19 \%$ male) was similar to the Balanced-towardOthers group except for higher social approach motivation, lower selfevaluations, higher inclusion of others in the self, and higher otherdirected emotions, thus reflecting a strong orientation toward others. Thus, this group was labeled as the Toward-Others Orientation. The fifth profile ( $n=317,40.3 \%$ of the sample, $35 \%$ male) was characterized by social motivation conflict, the highest level of unsociability, moderate to low self-evaluations, moderate inclusion of the others in the self, slightly higher emotional dysregulation, and lower than average levels of other-directed emotions. Thus, this orientation group appears to be the most conflicted in going both away and toward others, and was labeled the Conflicted-toward-Others Orientation.

\section{Distal outcomes}

Next, the model compared mean scores across orientation profiles on relational and individual distal outcomes. As noted previously, the Away Orientation group was small, and thus caution should be used in interpreting comparisons with this group. Group comparisons for relational and individual outcomes are presented in Figs. 2 and 3, respectively. Briefly summarized, results showed that individuals in the
Toward $(M=4.22, S E=.11)$ and Balanced $(M=3.98, S E=.09)$ Orientation groups had the highest relationship quality levels with best friends. In terms of romantic partner relationship quality, the Toward Orientation group $(M=4.40, S E=.15)$ reported the highest, followed by the Balanced group $(M=3.82, S E=.16)$. The Balanced $\left(M_{\text {mom }}=4.07, \quad S E_{\text {mom }}=.07, \quad M_{\text {dad }}=3.55, \quad S E_{\text {dad }}=.10\right)$, Toward $\left(M_{\text {mom }}=3.74, S E_{\text {mom }}=.11, M_{\text {dad }}=3.35, S E_{d a d}=.11\right)$, and Conflicted $\left(M_{\text {mom }}=3.61, S E_{\text {mom }}=.06, M_{\text {dad }}=3.17, S E_{\text {dad }}=.07\right)$ Orientation groups had higher levels of parental relationship quality, followed by the Dependent group $\left(M_{\text {mom }}=3.25, S E_{\text {mom }}=.13, \quad M_{\text {dad }}=2.86\right.$, $\left.S E_{\text {dad }}=.13\right)$, and then the Away group $\left(M_{\text {mom }}=2.53, S E_{\text {mom }}=.34\right.$, $\left.M_{\text {dad }}=1.90, S E_{d a d}=.37\right)$. In terms of depression, the Balanced Orientation $(M=1.28, S E=.04)$ demonstrated the best adjustment (i.e., lowest levels), with the Dependent Orientation group $(M=1.88$, $S E=.06)$ faring the worst. Lastly, the Away Orientation group ( $M=2.41, S E=.35)$ demonstrated the highest levels of substance use, followed by the Toward $(M=1.68, S E=.06)$ and Conflicted $(M=1.56, S E=.04)$.

\section{Discussion}

The current study aimed to first explore whether or not meaningful social orientation groups could be identified in emerging adults based on a number of social factors, and second, determine whether or not these social orientation groups are associated with varying levels of relational and individual well-being. Results suggest the existence of five different social orientations that relate to varying degrees of wellbeing. The findings from this study have important implications for research and intervention concerned with positive development and flourishing in emerging adulthood. Amidst the many personal and relational transitions during this time period, steady relationships are not only a sign of positive adjustment, but also help to foster other aspects of healthy development (Barry et al., 2016; Padilla-Walker et al., 2017). The social orientations perspective provides meaningful insight into some of the individual differences that contribute to these relationships. For example, some orientations (i.e., Balanced, Toward) may promote the formation of more relationships than other orientations (i.e., Dependent, Away). Additionally, the social orientations perspective also advances theory by presenting a novel and unifying framework to understand how emerging adults move toward and away from others, allowing for a more detailed discussion of social and nonsocial subtypes 


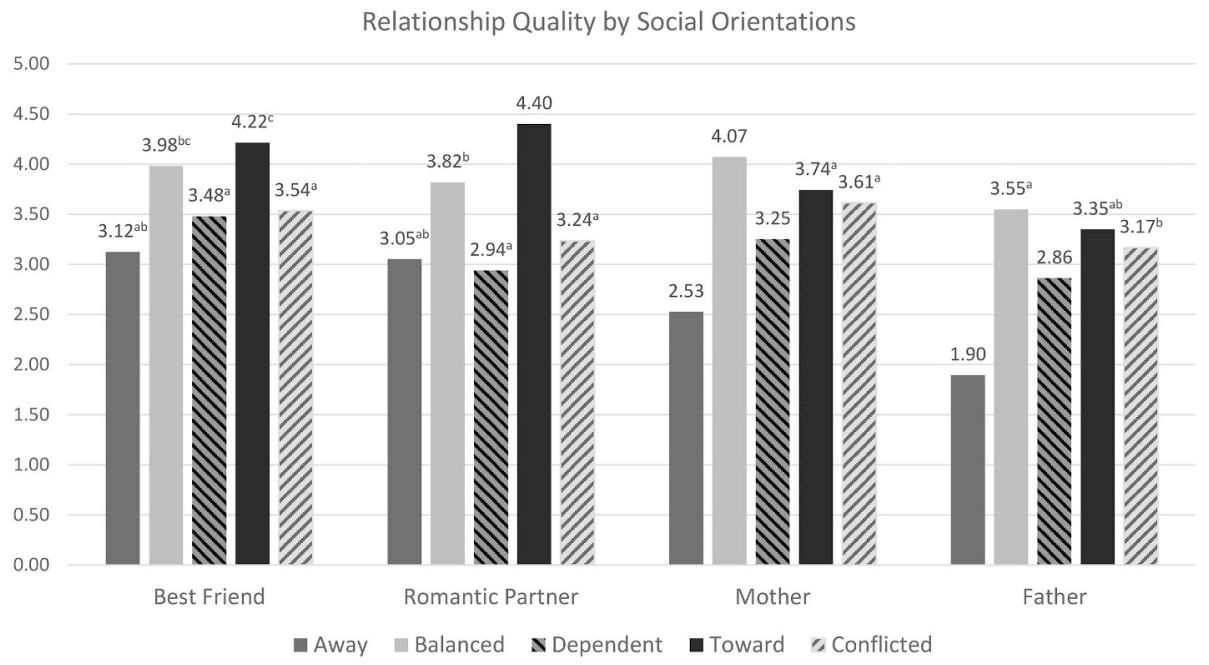

Fig. 2. Distal relational outcomes for each profile. Estimated means sharing a superscript letter are not statistically different at the $p<.05$ level.

and the nuances of how and why they relate differentially to well-being. Each social orientation is discussed in terms of its contribution to advancing theory as well as developmental and applied implications for emerging adulthood.

\section{Dependent toward others: too much other in the self}

The Dependent-toward-Others orientation group ( $n=86,10.9 \%$ of the sample, $23 \%$ male) displays an interesting albeit problematic combination of moving toward and away from others. They appear to move toward others with a desire for others' approval and positive feelings toward others, but are driven away from others by negative self-perceptions and oversensitivity to others' opinions. Given the many changes and transitions that take place during emerging adulthood, this social orientation is especially concerning for well-being and may be a target for intervention.

Indeed, it appears that people in the Dependent group are seeking the approval of others while perhaps doubting that they are worthy of this approval and fearing that it will not come. This may signify an unhealthy cycle in which desire for approval, personal insecurities, lack of close relationships, and depressive symptoms reinforce and exacerbate the situation. Specifically, these individuals may seek approval from others because they do not approve of themselves. However, their low self-esteem and sensitivity to rejection hamper the skills necessary to establish and maintain close relationships (Fitzsimons \& Anderson, 2013), and when hopes and expectations for relationships are not met, depressive symptoms are frequently experienced (Chango, McElhaney, Allen, Schad, \& Marston, 2012). The desire to connect with others likely does not go away, however, and these individuals are likely to continue making attempts at relationships. These attempts, made by individuals who are insecure, depressed, and lacking relational skills, may actually elicit negative responses from others in social interactions (Forest \& Wood, 2012), which only serve to reinforce the fears and insecurities already held. Thus, ironically and sadly, for individuals who are Dependent-toward-others, their fear of the very thing they are seeking may actually move them away from others and contribute to their struggles with close relationships and depression.

Taken together, the social orientations perspective helps to identify a group of emerging adults (i.e., Dependent-toward-others) who may be at risk of floundering due to internalizing difficulties. Hence, in being able to identify this group of struggling emerging adults, the social orientations perspective can inform individuals, parents, and interventionists concerned with the well-being of emerging adults, specifically in understanding processes that may underlie struggles with close relationships and depression. Outreach and intervention aimed at improving relational skills and strengthening self-esteem may be

\section{Individual Outcomes by Social Orientations}

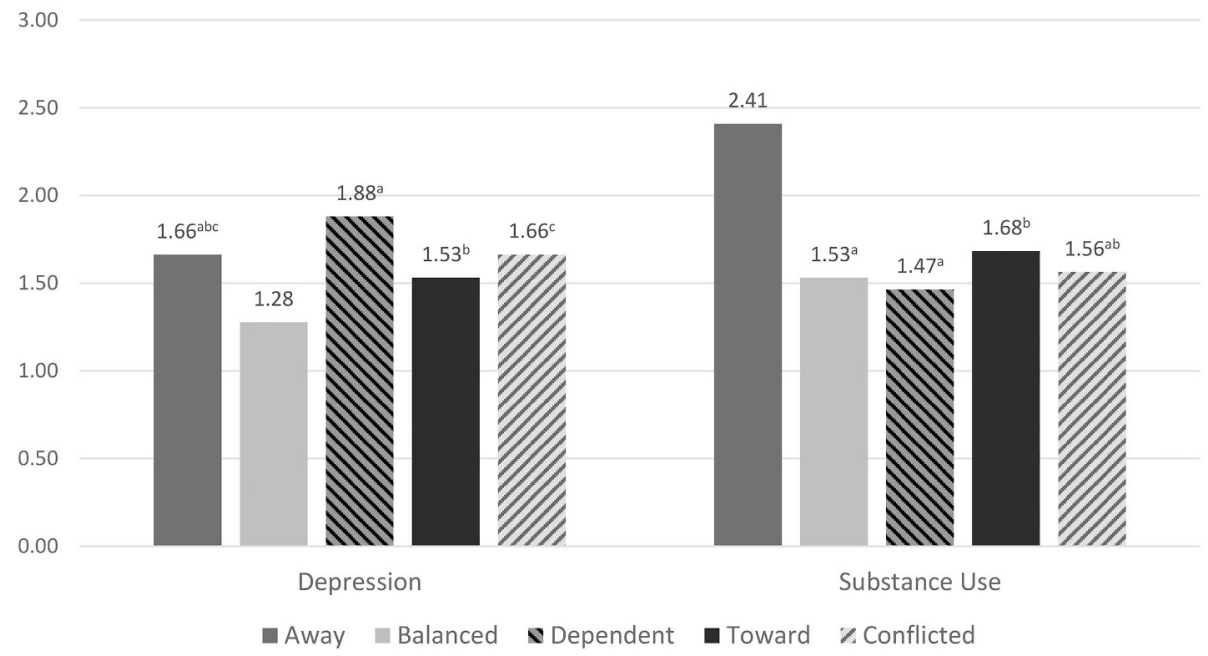

Fig. 3. Distal individual outcomes for each profile. Estimated means sharing a superscript letter are not statistically different at the $p<.05$ level. 
especially beneficial for emerging adults who are dependent-towardothers to establish a healthier balance between the self and others as they navigate the transitional period of emerging adulthood.

\section{Conflicted toward others: In between social and withdrawn}

The social orientations approach also provides a richer description of what it might mean to be unsociable, both challenging and adding to previous conceptualizations and suggesting that this newly identified group is common among emerging adults. The Conflicted-towardOthers group ( $n=317,40.3 \%$ of the sample, 35\% male) presents a unique combination of being oriented both toward and away from others. They are oriented toward others in a moderate inclusion of others in the self, positive regard for others (although empathic concern is lower than average in this group, based on the scale that was used the group mean still reflects positive regard for others), and moderate social involvement. Yet they are also oriented away from others in lower than average sociability, moderate shyness, and higher unsociability (i.e., preference for being alone). People in the Conflicted group are not simply low in social motivation, but rather demonstrate an interesting blend that can only be considered somewhere in between social and withdrawn, which is especially significant because nearly half of all emerging adults in this sample fit this description.

Several developmental trends support the finding that the Conflicted social orientation might be a common orientation found in emerging adults. During adolescence and emerging adulthood, inclusion of others in self-perceptions is normative and higher than at other developmental periods (Callan, Kim, \& Matthews, 2015; Harter, 2012), and thus the moderate inclusion of others found in the Conflicted group may be a normative trend, one that reflects more of an awareness of the thoughts of others rather than over-concern for others' opinions. Additionally, individuals often experience an increase of appreciation for time spent alone (i.e., unsociability) beginning in adolescence and continuing through adulthood (Goossens, 2014; Larson, 1997), which is consistent with the Conflicted group reporting higher levels of this characteristic. Results from this study further indicate that it may be normative for emerging adults to experience moderate social apprehension, positive thoughts and feelings toward others, and moderate social involvement, perhaps stemming from the novel and changing social contexts faced in emerging adulthood (e.g., college, work transitions, roommates). Overall, this orientation appears to be related to positive adjustment during emerging adulthood, but with some exceptions. Individuals in the Conflicted group are doing well with parental relationships and have low/average levels of substance use, but are less successful in best friendships and romantic relationships and experience moderate levels of depression. Again, these struggles may be due to the changing social contexts of emerging adulthood (Schulenberg, Sameroff, \& Cicchetti, 2004). The high level of reactivity reported in the Conflicted group may indicate that these individuals are easily overstimulated by novel or crowded social situations. Although they also demonstrate the ability to regulate intense emotions and appear to be fully capable of social interaction, they may be more passive in their interactions with others and less likely to actively pursue social interaction. As a result, Conflicted individuals may not meet as many friends or romantic partners as others or perhaps establish these relationships later than others (Boisvert \& Poulin, 2016). Yet because they are still prone to compare themselves to others, might understandably struggle with some level of depression (Buunk \& Gibbons, 2006). While the uncertainty and changes faced in emerging adulthood may present some challenges to those of this social orientation, these challenges may fade as people enter adulthood and face fewer changes to their social contexts. To summarize, findings related to the Conflicted social orientation are important because they identify a group of people not fully described by any previous study and indicate that this orientation is rather common among emerging adults.

\section{Away from others: implications for psychopathology}

It appears that those who have previously been labeled as socially avoidant (low approach, high avoidance) according to the motivational model (Asendorpf, 1990) might be those who fall into the Away-fromOthers orientation ( $n=12,1.5 \%$ of the sample, $75 \%$ male). By examining more than just social motivations, however, we get a much clearer picture of who these individuals are and why they may be so averse to social interactions. The Away-from-Others orientation is more than a simple motivational desire to be away from other people, but, rather, is based on a thorough cognitive, emotional, and behavioral detachment from others. Indeed, individuals in this group display markedly low concern for others (i.e., self in relation to others, otherdirected feelings) and moderate social involvement. By taking a holistic social orientations approach, this study makes a significant contribution by shedding new light on this group of individuals who are thoroughly oriented away from others and struggle on multiple indices of wellbeing. The results seem to raise concern regarding individuals who so strongly avert themselves away from others in all aspects of their lives, showing that that this orientation group is the most likely to struggle relationally and individually. In fact, the relatively extreme pattern of moving away from others and the small size of this group suggest that this orientation may be more pathological than normative. For example, the Away Orientation reflects aspects of social anhedonia (i.e., disinterest toward and lack of pleasure in social interaction; Bowker et al., 2017) and callous unemotional traits (i.e., disregard for others, lack of empathy; Frick \& White, 2008), although further work will be needed to explore these connections. Findings from this study also hint at possibilities of how this type of orientation might develop. For example, some characteristics of the Away-from-Others orientation, such as lack of empathy and avoidance of social information about the self, may be rooted in past experiences with social isolation (e.g., exclusion, rejection, ostracism; Twenge, Catanese, \& Baumeister, 2003). It is possible that people who are oriented away from others were previously oriented differently, but through repeated negative social experiences turned away from others. Much more work is needed to understand the developmental origins of this group, but the use of a social orientations approach may help us identify and, ultimately, better understand a small, but definitely at-risk, segment of emerging adults deserving of attention. Indeed, historically, this social group (i.e., avoidant individuals; Asendorpf, 1990) has received far less attention in research than others, and this study makes an important contribution by providing the most detailed description of them to date. These findings can motivate and inform future research that may be of particular interest to scholars and clinicians interested in psychopathology and peer exclusion in adolescence and emerging adulthood, as these appear to be closely related to being socially orientated away from others.

\section{Toward and balanced toward others: multiple forms of sociality}

Lastly, the social orientations approach also makes a significant contribution to our understanding of sociability. This study identified two distinct forms of sociability, Toward-Others $(n=171,21.7 \%$ of the sample, $19 \%$ male) and Balanced-toward-Others ( $n=201,25.5 \%$ of the sample, $34 \%$ male), whereas the social motivations model (Asendorpf, 1990) describes only one. Results underscore the importance of this distinction between types of sociability not only in the characteristics that describe them, but also in their differing levels of individual wellbeing. Of the two sociable groups, the Toward-Others orientation is characterized by higher social approach motivation, higher inclusion of others in self-perceptions, and higher other-directed emotions. This group also displays comparatively higher levels of depression and substance use than the Balanced group, indicating that although being oriented toward others may be good for interpersonal relationships, it may also pose some risks for individual well-being. The struggles of the 
Toward group may reflect being overly oriented toward others in some areas. Specifically, over concern for others and their opinions may make individuals more prone to depression and more likely to engage in substance use for social status and peer approval (Kashdan et al., 2008; Litt et al., 2015), whereas a balance between the self and others may protect against these risks. Hence, just as the traditional motivational model points at different ways of being withdrawn being associated with different outcomes, the social orientation approach identifies different ways of being social that are linked to various indices of relational and individual well-being. Specifically, this study shows that although an orientation toward others appears to be beneficial for relationships, the way in which people are oriented toward others makes a difference for individual outcomes. Just as an orientation completely away from others appears to be problematic, an orientation completely toward others may be likewise indicative of risk, albeit not to the same extent. This study is the first of its kind to not only suggest multiple forms of sociability in emerging adulthood, but also demonstrate that these are important for individual well-being.

Finally, a note should be made concerning the potential role of gender in the make-up of the groups. Specifically, the Away and Toward orientation groups were disproportionately comprised of men and women, respectively. The Away-from-Others orientation was comprised of $75 \%$ men, a strikingly high number, and the Toward orientation was comprised of $81 \%$ women. Conversely, the other profiles more closely reflected the overall sample of roughly $70 \%$ women. From the outset, gender was not a central component of the study because there was little in our initial review of literature or theory to cause us to expect the groups to vary based on gender. Furthermore, there was nothing in the analyses themselves that would allow us to speak definitively about why this might be the case. Hence, a post-hoc attempt to explain these differences will be purely speculative in nature. However, the gender socialization literature suggests that girls and women are particularly socialized to be attuned to relationships and the ethics of caring (Chodorow, 1989; Gilligan, 1982; Huston, 1983; Miller, 1986). Hence, female emerging adults may be socialized more strongly than male emerging adults to orient themselves toward others. The results of the current study underscore the level of individual differences across the numerous factors that make up a social orientation (i.e., social motivations, self-evaluations, other-directed emotions, etc.), but they may also reflect the results of gender socialization by pointing to differences between men and women in the extent to which awareness of and relationships with others factor into their orientations generally. Future work should be conducted with the purposeful intent of exploring gender differences in social orientations.

In summary, by taking a holistic approach to social orientations, rather than only examining social motivation, this study makes several important contributions to the field of social development. Specifically, this study identifies two social orientations that may be targets for intervention (i.e., Dependent and Away), discusses why most emerging adults may fall somewhere between sociability and withdrawal, and identifies two distinct forms of sociability each relating to varying degrees of relational and individual well-being.

\section{Limitations and future directions}

Despite the contributions this study makes, it is not without limitations. First, this sample of emerging adults did not include non-college students and is thus not representative of all emerging adults in the United States (Arnett, 2016). This may be an important consideration given the topic of social orientations. For example, an orientation away from others may drive people away from the highly social college atmosphere. Thus, it is possible that the number of people fitting into each orientation category will be different in the general population of emerging adults, or that there are entirely new types of orientations not observed in the present study. The sample also lacked racial and ethnic diversity, which may similarly limit conclusions about group sizes and types of social orientations. There is certainly reason to believe that cultural factors (e.g., collectivist views) would influence how people are oriented toward others (Ding et al., 2015), and this could be a fruitful avenue for future inquiry. Future work should examine social orientations in more diverse, representative samples to explore the possibility of more orientations and the potential role of culture.

Furthermore, the measures utilized in this study could have more thoroughly assessed the proposed aspects of social orientations. For example, the measures of self-regulation could be more focused on regulation specifically within social situations, and measures of social behavior could be broader to capture multiple types of social interaction (i.e., attending parties, engaging in conversations, etc.). Future improvement of measures could help with more precise classifications and descriptions of social orientation groups. Nonetheless, the measures used in this study do measure some important aspects of self-regulation and actual social behavior and, as such, did contribute to differentiation between groups.

Findings from this study point to a number of interesting avenues for future research to address, particularly in regard to understanding these social orientations from a developmental perspective. This includes exploration of the existence of different social orientations at various developmental periods. For example, many factors that contributed to social orientations in this study, such as self-consciousness and empathic concern, develop over time and are manifest differently at various developmental stages. Therefore, there may be additional or fewer social orientations that exist throughout childhood, adolescence, and adulthood. Also important is research on how social orientations develop and change over time for individuals, including the potential role of social experiences in shaping a person's orientation. For example, individuals subject to frequent social rejection display many similarities to the Away-from-Others orientation, such as lack of empathizing with others, emotional insensitivity, impaired self-regulation, decreased self-esteem, and seeking to escape social information about the self to avoid painful reflection on personal flaws (Twenge et al., 2003). Hence, peer rejection may be an important factor in one's developing orientation toward or away from others. In sum, it is possible that social experiences, positive or negative, can shape and change how individuals are oriented toward others, and future work should identify what these experiences are and the impact they can have.

\section{Conclusion}

Despite these limitations, however, this study offers a significant contribution to current models of social development and interaction. This is the first study of its kind to assess social orientations from a holistic, person-centered approach, identifying five meaningful social orientations that differ in terms of relational and individual well-being. In doing so, the results suggest that balancing the connections between others and the self leads to optimal relational and individual outcomes, whereas moving further toward or away from others comes with fewer benefits and increased risks. Findings from this study are meaningful not only to researchers, but also provide helpful perspectives to practitioners (especially those in university settings), parents, and adolescents and emerging adults themselves. As this is the first study to consider multiple aspects of social orientations, it provides one of the clearest and most detailed descriptions of social subtypes in emerging adulthood to date.

\section{Appendix A. Supplementary data}

Supplementary data to this article can be found online at https:// doi.org/10.1016/j.appdev.2018.09.001.

\section{References}

Arnett, J. J. (2016). Introduction: Emerging adulthood theory and research: Where we are 
and where we should go. In J. Arnett (Ed.). The Oxford handbook of emerging adulthood (pp. 1-7). New York, NY: Oxford University Press.

Aron, A., Aron, E. N., \& Smollan, D. (1992). Inclusion of other in the self scale and the structure of interpersonal closeness. Journal of Personality and Social Psychology, 63 596-612.

Asendorpf, J. B. (1990). Beyond social withdrawal: Shyness, unsociability, and peer avoidance. Human Development, 33, 250-259.

Asparouhov, T., \& Muthén, B. (2014). Auxiliary variables in mixture modeling: Three-step approaches using Mplus. Structural Equation Modeling: A Multidisciplinary Journal, 21, 329-341.

Barry, C. M., Madsen, S. D., \& DeGrace, A. (2016). Growing up with a little help from their friends in emerging adulthood. In J. Arnett (Ed.). The Oxford handbook of emerging adulthood (pp. 215-229). New York, NY: Oxford University Press.

Batanova, M. B., \& Loukas, A. (2011). Social anxiety and aggression in early adolescents: Examining the moderating roles of empathic concern and perspective taking. Journal of Youth and Adolescence, 40, 1534-1543.

Baumeister, R. F., \& Leary, M. R. (1995). The need to belong: Desire for interpersonal attachments as a fundamental human motivation. Psychological Bulletin, 117 497-529.

Baumeister, R. F., \& Vohs, K. D. (2012). Self-regulation and the executive function of the self. In M. Leary, \& J. Tangney (Eds.). Handbook of self and identity (pp. 180-197). (2nd ed.). New York, NY: The Guilford Press.

Berger, C., Batanova, M., \& Cance, J. D. (2015). Aggressive and prosocial? Examining latent profiles of behavior, social status, Machiavellianism, and empathy. Journal of Youth and Adolescence, 44, 2230-2244.

Boisvert, S., \& Poulin, F. (2016). Romantic relationship patterns from adolescence to emerging adulthood: Associations with family and peer experiences in early adolescence. Journal of Youth and Adolescence, 45, 945-958.

Bowker, J. C., Nelson, L. J., Markovic, A., \& Luster, S. (2014). Social withdrawal during adolescence and emerging adulthood. In R. Coplan, \& J. Bowker (Eds.). The handbook of solitude: Psychological perspectives on social isolation, social withdrawal, and being alone (pp. 167-183). New York, NY: Wiley-Blackwell.

Bowker, J. C., Stotsky, M. T., \& Etkin, R. G. (2017). How BIS/BAS and psycho-behavioral variables distinguish between social withdrawal subtypes during emerging adulthood. Personality and Individual Differences, 119, 283-288.

Buunk, A. P., \& Gibbons, F. X. (2006). Social comparison orientation: A new perspective on those who do and those who don't compare with others. In S. Guimond (Ed.) Social comparison and social psychology: Understanding cognition, intergroup relations and culture (pp. 15-32). New York, NY: Cambridge University Press.

Carbery, J., \& Buhrmester, D. (1998). Friendship and need fulfillment during three phases of young adulthood. Journal of Social and Personal Relationships, 15, 393-409.

Callan, M. J., Kim, H., \& Matthews, W. J. (2015). Age differences in social comparison tendency and personal relative deprivation. Personality and Individual Differences, 87, 196-199.

Chango, J. M., McElhaney, K. B., Allen, J. P., Schad, M. M., \& Marston, E. (2012). Relational stressors and depressive symptoms in late adolescence: Rejection sensitivity as a vulnerability. Journal of Abnormal Child Psychology, 40, 369-379.

Cheek, J. M., \& Buss, A. H. (1981). Shyness and sociability. Journal of Personality and Social Psychology, 41, 330-339.

Chodorow, N. J. (1989). Feminism and psychoanalytic theory. New Haven, CT: Yale University Press.

Coplan, R. J., \& Armer, M. (2007). A "multitude" of solitude: A closer look at social withdrawal and nonsocial play in early childhood. Child Development Perspectives, 1, $26-32$.

Coplan, R. J., Prakash, K., O'Neil, K., \& Armer, M. (2004). Do you "want" to play? Distinguishing between conflicted shyness and social disinterest in early childhood. Developmental Psychology, 40, 244-258.

Davis, M. H. (1983). The effects of dispositional empathy on emotional reactions and helping: A multidimensional approach. Journal of Personality, 51, 167-184.

Ding, X., Coplan, R. J., Sang, B., Liu, J., Pan, T., \& Cheng, C. (2015). Young Chinese children's beliefs about the implications of subtypes of social withdrawal: A first look at social avoidance. British Journal of Developmental Psychology, 33, 159-173.

Eisenberg, N., Fabes, R. A., \& Murphy, B. C. (1995). Relations of shyness and low sociability to regulation and emotionality. Journal of Personality and Social Psychology, $68,505-517$.

Etkin, R. G., Bowker, J. C., \& Scalco, M. D. (2016). Associations between subtypes of social withdrawal and emotional eating during emerging adulthood. Personality and Individual Differences, 97, 239-244.

Farley, J. P., \& Kim-Spoon, J. (2014). The development of adolescent self-regulation: Reviewing the role of parent, peer, friend, and romantic relationships. Journal of Adolescence, 37, 433-440.

Feingold, A., Tiberio, S. S., \& Capaldi, D. M. (2014). New approaches for examining as sociations with latent categorical variables: Applications to substance abuse and aggression. Psychology of Addictive Behaviors, 28, 257-267.

Fitzsimons, G. M., \& Anderson, J. E. (2013). Interpersonal cognition: Seeking, understanding, and maintaining relationships. In D. Carlston (Ed.). Oxford handbook of social cognition (pp. 590-615). New York, NY: Oxford University Press.

Forest, A. L., \& Wood, J. V. (2012). When social networking is not working: Individuals with low self-esteem recognize but do not reap the benefits of self-disclosure on Facebook. Psychological Science, 23, 295-302.
Frick, P. J., \& White, S. F. (2008). Research review: The importance of callous-unemotional traits for developmental models of aggressive and antisocial behavior. Journal of Child Psychology and Psychiatry, 49, 359-375.

Gilligan, C. (1982). In a different voice: Psychological theory and women's development Cambridge, MA: Harvard University Press.

Goossens, L. (2014). Affinity for aloneness in adolescence and preference for solitude in childhood: Linking two research traditions. In R. Coplan, \& J. Bowker (Eds.). The handbook of solitude: Psychological perspectives on social isolation, social withdrawal, and being alone (pp. 150-166). New York, NY: Wiley-Blackwell.

Hart, E. A., Leary, M. R., \& Rejeski, W. J. (1989). The measurement of social physique anxiety. Journal of Sport and Exercise Psychology, 11, 94-104.

Harter, S. (2012). The construction of the self: Developmental and sociocultural foundations (2nd ed.). New York, NY: The Guilford Press.

Huston, A. C. (1983). Sex-typing. E. M. Hetherington (Vol. ed.) In P. Mussen (Ed.). Handbook of child psychology: Vol. 4. Socialization, personality, and social development (pp. 387-467). (4th ed.). New York, NY: Wiley.

Kashdan, T. B., Elhai, J. D., \& Breen, W. E. (2008). Social anxiety and disinhibition: An analysis of curiosity and social rank appraisals, approach-avoidance conflicts, and disruptive risk-taking behavior. Journal of Anxiety Disorders, 22, 925-939.

Kashdan, T. B., McKnight, P. E., Richey, J. A., \& Hofmann, S. G. (2009). When social anxiety disorder co-exists with risk-prone, approach behavior: Investigating a neglected, meaningful subset of people in the National Comorbidity Survey-Replication. Behaviour Research and Therapy, 47, 559-568.

Laible, D. J., Murphy, T. P., \& Augustine, M. (2014). Adolescents' aggressive and prosocial behaviors: Links with social information processing, negative emotionality, moral affect, and moral cognition. The Journal of Genetic Psychology, 175, 270-286.

Lanza, S. T., Tan, X., \& Bray, B. C. (2013). Latent class analysis with distal outcomes: A flexible model-based approach. Structural Equation Modeling, 20, 1-26.

Larson, R. W. (1997). The emergence of solitude as a constructive domain of experience in early adolescence. Child Development, 68, 80-93.

Leary, M. R. (1983). Social anxiousness: The construct and its measurement. Journal of Personality Assessment, 47, 66-75.

Leary, M. R., \& Tangney, J. P. (2012). The self as an organizing construct in the behavioral and social sciences. In M. Leary, \& J. Tangney (Eds.). Handbook of self and identity (pp. 1-18). (2nd ed.). New York, NY: The Guilford Press.

Litt, D. M., Stock, M. L., \& Gibbons, F. X. (2015). Adolescent alcohol use: Social comparison orientation moderates the impact of friend and sibling behaviour. British Journal of Health Psychology, 20, 514-533.

Miller, J. B. (1986). Toward a new psychology of women (2nd ed.). Boston, MA: Beacon Press.

Neeman, J., \& Harter, S. (1986). Manual for the Self-Perception Profile for College Students. Unpublished manuscriptDenver, Colorado: University of Denver.

Nelson, L. J. (2013). Going it alone: Comparing subtypes of withdrawal on indices of adjustment and maladjustment in emerging adulthood. Social Development, 22, $522-538$.

Nelson, L. J., Coyne, S. M., Howard, E., \& Clifford, B. N. (2016). Withdrawing to a virtual world: Associations between subtypes of withdrawal, media use, and maladjustmen in emerging adults. Developmental Psychology, 52, 933-942.

Nelson, L. J., \& Padilla-Walker, L. M. (2013). Flourishing and floundering in emergingadult college students. Emerging Adulthood, 1, 67-78.

Novak, S. P., \& Clayton, R. R. (2001). The influence of school environment and selfregulation on transitions between stages of cigarette smoking: A multilevel analysis. Health Psychology, 20, 196-207.

Nylund, K. L., Asparouhov, T., \& Muthén, B. O. (2007). Deciding on the number of classes in latent class analysis and growth mixture modeling: A Monte Carlo simulation study. Structural Equation Modeling, 14, 535-569.

Padilla-Walker, L. M., Memmott-Elison, M. K., \& Nelson, L. J. (2017). In L. PadillaWalker, \& L. Nelson (Eds.). Flourishing in emerging adulthood: Positive development during the third decade of life (pp. 212-236). New York, NY: Oxford University Press Positive relationships as an indicator of flourishing during emerging adulthood.

Radloff, L. S. (1977). The CES-D scale: A self-report depression scale for research in the general population. Applied Psychological Measurement, 1, 385-401.

Schulenberg, J. E., Sameroff, A. J., \& Cicchetti, D. (2004). The transition to adulthood as a critical juncture in the course of psychopathology and mental health. Development and Psychopathology, 16, 799-806.

Shulman, S., \& Connolly, J. (2016). In J. Arnett (Ed.). The challenge of romantic relationships in emerging adulthood (pp. 230-244). New York, NY: Oxford University Press The Oxford handbook of emerging adulthood.

Tein, J. Y., Coxe, S., \& Cham, H. (2013). Statistical power to detect the correct number of classes in latent profile analysis. Structural Equation Modeling, 20, 640-657.

Twenge, J. M., Catanese, K. R., \& Baumeister, R. F. (2003). Social exclusion and the deconstructed state: Time perception, meaninglessness, lethargy, lack of emotion, and self-awareness. Journal of Personality and Social Psychology, 85, 409-423.

Wills, T. A., Walker, C., Mendoza, D., \& Ainette, M. G. (2006). Behavioral and emotional self-control: Relations to substance use in samples of middle and high school students. Psychology of Addictive Behaviors, 20, 265-278.

Yang, C. (2016). Instagram use, loneliness, and social comparison orientation: Interact and browse on social media, but don't compare. Cyberpsychology, Behavior and Social Networking, 19, 703-708. 